\title{
Simultaneous laparoscopic adrenalectomy and laparoscopic nephron-sparing surgery - new experience with port placement
}

\author{
Wojciech Panek ${ }^{1}$, Tomasz Szydelko ${ }^{1,2}$, Jaroslaw Lewandowski ${ }^{1}$, Tomasz Tuchendler ${ }^{1}$, Grzegorz Urbańczyk ${ }^{1}$, Adam Litarski ${ }^{1}$, \\ Wojciech Apoznański ${ }^{3}$ \\ ${ }^{1}$ Clinical Department of Urology, $4^{\text {th }}$ Clinical Military Hospital, Wroclaw, Poland \\ 2Department of Palliative Care Nursing, Wroclaw Medical University, Wroclaw, Poland \\ ${ }^{3}$ Department of Pediatric Surgery and Urology, Wroclaw Medical University, Wroclaw, Poland
}

Videosurgery Miniinv 2013; 8 (4): 357-360

DOI: 10.5114/wiitm.2011.35351

\begin{abstract}
The aim of the study was to describe simultaneous laparoscopic adrenalectomy and laparoscopic nephron-sparing surgery, to discuss the details of a convenient laparoscopic approach and the way of port placement, as well as to present a review of the literature concerning combined laparoscopic procedures. A 72-year-old woman was admitted to our department because of a tumor of the right adrenal gland and a small tumor of the right kidney. The patient underwent simultaneous laparoscopic adrenalectomy and laparoscopic nephron-sparing surgery. The postoperative period was uncomplicated. The patient was discharged from the hospital on the $4^{\text {th }}$ postoperative day. We believe that the proposed way of trocar placement would help to avoid a 'rollover' problem between the laparoscope and a Satinsky clamp or a 'crossing swords' problem between a Satinsky clamp and manipulators.
\end{abstract}

Key words: laparoscopic adrenalectomy, laparoscopic NSS, simultaneous management.

\section{Introduction}

Adrenal tumors are found in $0.35-4.4 \%$ of abdominal computed tomography (CT) scans and in up to $9 \%$ of autopsy studies [1]. The identification of adrenal masses among patients evaluated with ultrasound for non-endocrinological complaints ranges from $0.4 \%$ to $1.9 \%$ [2].

The indications for surgical management of adrenal tumors include secretory tumors (pheochromocytomas, glucocorticoid secreting adrenal tumors, primary aldosteronism), adrenocortical carcinoma, adrenal metastases and asymptomatic, biochemically inert masses larger than $4 \mathrm{~cm}$ or smaller than $4 \mathrm{~cm}$ but enlarging in radiological examinations during the follow-up. With its minimally invasive nature, good access into the operating area, short hospital stay, small blood loss, satisfactory cosmetic result and early return to normal activity, laparoscopic adrenalectomy is considered a gold standard in the treatment of adrenal tumors [3].

Increasing incidence of small renal masses (particularly with size from $2 \mathrm{~cm}$ to $4 \mathrm{~cm}$ ) has been observed for the last decades (from 1.0 case per 100,000 in 1983 to 3.3 cases per 100,000 in 2002) [4]. The standard surgical option for these cT1a tumors is nephron-sparing surgery (NSS). A laparoscopic approach in cases with favorable tumor locations is an alternative to an open procedure in experienced hands.

In this report, we present a coincidence of two lesions - a small renal tumor and an adrenal tumor 
on the ipsilateral side - which created an opportunity to perform a combined laparoscopic procedure.

The aim of the study was to describe simultaneous laparoscopic adrenalectomy and laparoscopic nephron-sparing surgery, to discuss the details of a convenient laparoscopic approach and the way of port placement, as well as to present a review of the literature concerning combined laparoscopic procedures.

\section{Case report}

A 72-year-old woman was admitted to our department due to a hormonally active, cortisol producing tumor of the right adrenal gland and a small tumor of the right kidney revealed during the search for the cause of hypertension in the Endocrinological Department. On admission, she remained in good general condition, without relevant complaints. She suffered from arterial hypertension (treated with Indapamide $1.25 \mathrm{mg}$ orally), hypothyroidism (treated with Levothyroxine $50 \mathrm{mg}$ orally) and gastritis. Her past medical history also included open cholecystectomy in 1981 and ischemic cerebral stroke 13 years later with right hemiparesis. Physical examination was unremarkable except for hemiparesis. Laboratory tests, X-ray chest examination and electrocardiogram performed on admission were correct. Abdominopelvic CT scans showed a tumor of the right adrenal gland (45 $\mathrm{mm} \times$

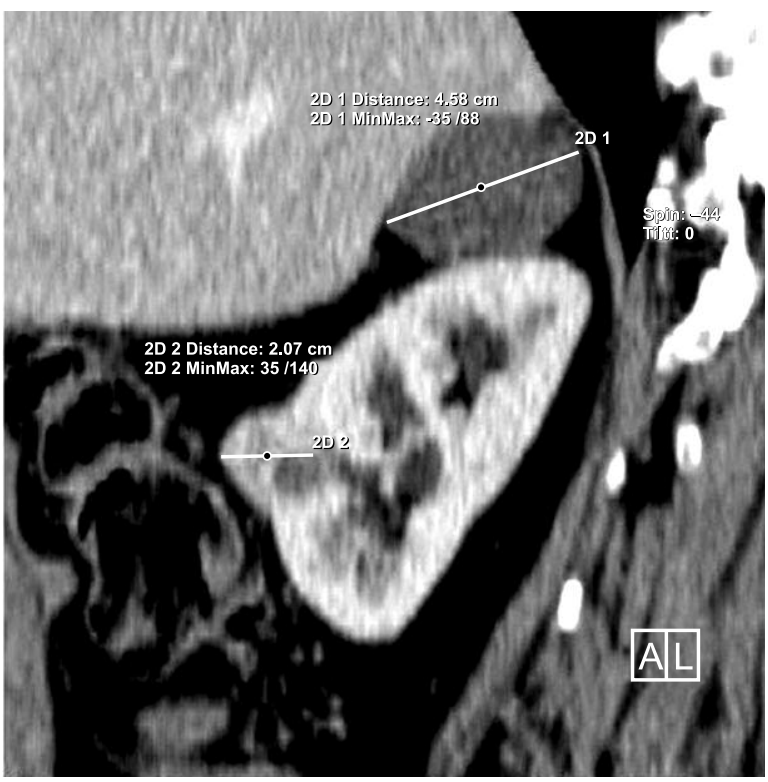

Photo 1. Computed tomography showing an adrenal tumor and a small renal tumor
$41 \mathrm{~mm} \times 28 \mathrm{~mm})$ and a renal tumor $(22 \mathrm{~mm} \times 24 \mathrm{~mm}$ $\times 22 \mathrm{~mm}$ ) presenting good contrast enhancement, located on the anterior surface of the right kidney in the middle portion of the organ (Photo 1). Simultaneous laparoscopic adrenalectomy and laparoscopic nephron-sparing surgery (NSS) were performed after intravenous perioperative antibiotic prophylaxis. In the postoperative period Hydrocortisone $(4 \times 50 \mathrm{mg}$ i.v.) was administered.

\section{Surgical technique}

The patient was operated on under general anesthesia. Before the procedure, the patient was catheterized and placed in a lateral supine position. A Hasson $2 \mathrm{~cm}$ minilaparotomy below the umbilicus was made to create pneumoperitoneum. Two $5 \mathrm{~mm}$ trocars were placed under direct vision just below the costal margin: laterally to the rectus muscle and in the anterior axillary line. The fourth trocar was placed in the mid-clavicular line at the level of the umbilicus (Figure 1). After releasing the hepatic flexure, the right hemicolon was mobilized and dislocated medially, entering the right retroperitoneal space. The loop of the duodenum was dislocated medially and the vena cava was identified. The short adrenal vein was

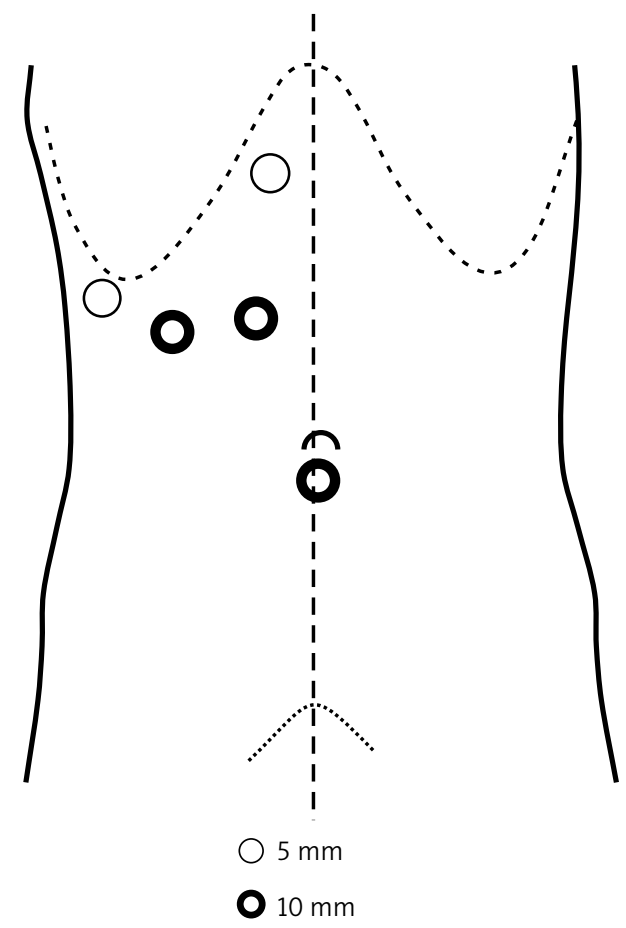

Figure 1. Trocar placement 
identified, clipped and transected. A LigaSure device (Tyco Healthcare UK Ltd., Gosport, UK) was used to safely mobilize the whole gland. After this, a specimen was put into a laparoscopic bag.

Before NSS was started, an additional $10 \mathrm{~mm}$ trocar was placed on the lateral edge of the right rectus muscle cephalad to a $10 \mathrm{~mm}$ trocar located below the umbilicus. This additional trocar was used for the laparoscope and a Satinsky clamp was inserted through the trocar located below the umbilicus (previously used for the laparoscope during adrenalectomy). The renal vein and artery were dissected and prepared for clamping. The tumor was disclosed after an incision of the perinephric adipose tissue; the fat tissue covering the tumor was resected and entrapped in a laparoscopic bag. Before the clamping of the renal vessels, $125 \mathrm{ml}$ of $20 \%$ mannitol solution was injected intravenously. After the clamping of the renal vein and artery, tumorectomy was performed and the renal capsule was sewn with a running suture. To minimize bleeding, TachoSil (Nycomed Austria $\mathrm{GmbH}$ ) was used. Before releasing the Satinsky clamp, $125 \mathrm{ml}$ of $20 \%$ mannitol solution was injected intravenously again. The time of warm ischemia of the kidney lasted 17 min. The tumor was put into the laparoscopic bag together with the fat tissue and adrenal gland and the bag was extracted through the widened incision below the umbilicus. Hemostasis was satisfactory at the end of the procedure. A closed suction drain was inserted through the $5 \mathrm{~mm}$ trocar and positioned near the kidney. Total blood loss was negligible and the total operative time was 205 min. The postoperative period was uncomplicated. The urinary catheter was removed on the second postoperative day and the suction drain was removed 1 day later. Supplementation of Hydrocortisone in decreasing doses was finished on the second postoperative day. The patient was discharged in general good condition on the $4^{\text {th }}$ postoperative day.

Histological examination revealed benign cortical adenoma ( $4.5 \mathrm{~cm}$ in diameter) of the right adrenal gland and renal cell carcinoma (clear cell type) of the right kidney $(1.2 \mathrm{~cm}$ in diameter, pT1a). There was no cancerous infiltration of the fat tissue covering the tumor.

\section{Discussion}

There are not many studies concerning laparoscopic adrenalectomy combined with other laparoscopic procedures in the literature. Gupta et al. described successful simultaneous laparoscopic adrenalectomy for pheochromocytoma and pyeloplasty for uretero-pelvic junction obstruction [5]. Castillo et al. described laparoscopic excision of the left adrenal gland due to a $5 \mathrm{~cm}$ mass with simultaneous partial resection of a cystic lesion found on the tail of the pancreas in a patient with von Hippel-Lindau disease [6]. Schwartz presented three cases of laparoscopic adrenalectomies combined with simultaneous renal surgery: nephrectomy, renal cyst decortication and renal cryotherapy [1].

Maurya et al. analyzed retrospectively the outcomes of multiple laparoscopic procedures performed in one centre. The aim of their study was to analyze the morbidity after simultaneous laparoscopic operations. The patients were divided into three groups according to the kind of procedures performed: laparoscopic adrenalectomy with another laparoscopic procedure (group 1), laparoscopic nephrectomy with another laparoscopic procedure (group 2) and laparoscopic procedures involving the renal pelvis/ureter combined with another procedure (group 3). The mean operative time, blood loss, hospital stay and expenditure in the three groups were analyzed. Only the mean operative time and mean hospital stay were significantly prolonged in group 1 [7].

Tsivian et al. combined laparoscopic renal surgery with laparoscopic cholecystectomy and in 1 case they added a third laparoscopic procedure (a bilateral salpingo-oophorectomy) [8]. Romero et al. described 2 cases of right laparoscopic radical nephrectomy and simultaneous laparoscopic excision of a liver tumor [9].

All the papers mentioned above seem to indicate that combined laparoscopic procedures are feasible and they are not connected with a high complication rate. We believe that in experienced hands they constitute an attractive alternative to open operations.

Typical port placement for laparoscopic nephronsparing surgery is similar to the approach for nephrectomy: a $10 \mathrm{~mm}$ trocar is placed below the umbilicus (for the laparoscope), a $12 \mathrm{~mm}$ trocar is placed laterally to the rectus muscle at the level of the umbilicus (for instrumentation and stapler), and one or two $5 \mathrm{~mm}$ trocars are placed in different locations [10]. However, the above port placement can be applied when 'bulldog' clamps are used for vascular clamping or in patients with small, peripheral masses, where a "non-clamping" technique can be used 
[11]. The authors use the Satinsky clamp because it does not force a precise and separate dissection of the renal vessels. Moreover, the release of the Satinsky clamp is quick and technically easy. In contrast, release of a 'bulldog' clamp requires a precise grasp with a dedicated instrument. Therefore it is sometimes difficult and time-consuming, hence prolonging the time of warm ischemia. On the other hand, the use of the Satinsky clamp requires an additional port, which may lead to 'rollover' and 'crossing swords' problems. It seems to us that our proposal of port placement provides optimal access of the Satinsky clamp to the renal vessels without disturbing the movements of the laterally placed laparoscope and manipulators and makes the combined procedure comfortable.

In conclusion, optimal positioning of trocars is an important aspect of successful performance of the laparoscopic procedure. Our proposition of laparoscopic access in the case of coexisting adrenal and renal pathologies may be useful for surgeons who plan to perform combined laparoscopic procedures.

\section{References}

1. Schwartz BF. Laparoscopic adrenalectomy with simultaneous management of renal pathology. BJU International 2004; 93; 417-9.

2. Aron D, Terzolo M, Cawood TJ. Adrenal incidentalomas. Best Pract Res Clin Endocrinol Metab 2012; 26: 69-82.

3. Szydełko T. Atlas of laparoscopic urology. Chtosta P, Stojewski M (eds). Polish Association of Urology, Warsaw 2008; 67-76.

4. Hollingsworth JM, Miller CD, Daignault S, Hollenbeck BK. Rising incidence of small renal tumor: a need to reassess treatment effect. J Natl Cancer Inst 2006; 98: 1331-4.

5. Gupta NP, Kumar R, Tandon S. Simultaneous laparoscopic adrenalectomy for pheochromocytoma and dismembered pyeloplasty for uretero-pelvic junction obstruction. I Laparoendosc Adv Surg Tech A 2005; 15: 405-7.

6. Castillo O, Kerkebe M, Vitagliano G, Arellano L. Single-stage laparoscopic adrenalectomy and pancreatic cyst exsicion in a patient with von Hippel-Lindau disease. Actas Urol Esp 2007; 31: 292-4.

7. Maurya K, Sivanandam SE, Sukumar S, et al. Concomitant laparoscopic urological procedures: does it contribute to morbidity? J Minim Access Surg 2009; 5: 67-71.

8. Tsivian A, Konstantinovsky A, Tsivian M, et al. Concomitant laparoscopic renal surgery and cholecystectomy: outcomes and technical considerations. J Endourol 2009; 23: 1839-42.

9. Romero FR, Wagner AA, Bagga HS, et al. Laparoscopic treatment of simultaneous tumors in the liver and kidney. Urol Int 2007; 79: 142-4.
10. Bishoff JT, Kavoussi LR. Laparoscopic surgery of the kidney. In: Campbell's urology. Wein AJ, Kavoussi LR, Novick AC, et al. (eds). Saunders, Philadelphia 2007; 1759-809.

11. Petrasz P, Stojewski M, Sikorski A. Impact of "non-clamping technique" on intra- and postoperative course after laparoscopic partial nephrectomy. Videosurgery Miniinv 2012; 7: 275-9.

Received: 16.01.2013, accepted: 25.03.2013. 